\title{
From Collegial to Collaborative The Long Road to Building a Sustainable and Standardized Research Technology Service
}

\author{
Gary L. Pratt, Chief Information Officer \\ Kansas State University
}

S

etting the Stage

Kansas State University (KSU - K-State), founded in 1863, is the nation's first operational Land Grant University. It is home to 24,000 students and 1,400 faculty, with over 250 undergraduate majors and programs and 160+ graduate degrees and certificates. Five years ago, KSU published a visionary strategic plan with very aspirational goals for 2025.

K-State 2025 Visionary Plan states as its first theme: "Research, Scholarly and Creative Activities, and Discovery (RSCAD)" - with a thematic goal: "Create a culture of excellence that results in flourishing, sustainable, and widely recognized research, scholarly and creative activities, and discovery in a variety of disciplines and endeavors that benefit society as a whole." Ultimately, K-State aspires to reach the goal of becoming a top 50 research university (Kansas State University. (2011). K-State 2025: A Visionary Plan for Kansas State University).

In October 2017, I started as the Chief Information Officer (CIO) for KState. The CIO position, in the past reported to the Provost. A new President chose to change that reporting structure so that the CIO reported to him. The President, General Richard Myers, was in 2001 the Chairman of the Joint Chiefs of Staff reporting to President Bush during the 9-11 terrorist attacks - the highest ranking military officer in the United States leading the response to these horrible events. He not only understands the business of information technology (IT), he led cyber-security at the national level, and knows what it means to make IT strategic to the organization. ... no pressure.

Once coming to Kansas State University, I began a listening tour, talking with hundreds of individuals from across multiple campuses (students, staff, faculty, governance groups, and executives) to learn what was working, what was not working, and what we should be doing that we currently were not doing in IT. As expected, I heard a lot. ... A LOT!

To add to the complexity of the KState IT environment, $50 \%$ of the institution's 300+ information technology staff do not report to me (central IT). There are over 30 islands of IT setting their own direction, implementing their own standards, running their own enterprise applications, and on and on.

See any potential for issues? This environment led to many examples of duplicated, sometimes competing sys- 
tems. There were no formal sets of standards for providing services, leaving significant concerns, including a lot of "haves" and even more "have-nots", few economies of scale for purchasing, quite a few blind sides for needs of support, the potential for issues with security, and users left on their own to figure out how to get help.

Having done this at three other higher education institutions, I knew that I could probably write an IT Strategic Plan based upon the information I learned on this multi-month listening tour. However, I also knew that doing this would make a plan based on the world according to Gary. Although well focused and aligned with the needs I heard expressed, this is not the way to create buy-in. I had to run a formal strategic planning process.

I moved quickly to a formal procurement process and brought in an outside consultant. My leadership team worked with this consultant to develop script drafts for different constituent groups; students, staff, and faculty that represented various business and academic units across K-State. Once finalized, we had the consultant use these scripts to perform formal information gathering sessions (focus groups, keystakeholder interviews, and a web survey) getting participation and valuable input from well over 250 students, faculty, and staff face-to-face and 1,300 individuals through the web survey.

\section{What Did I Find?}

"A series of cottage industries brought together by a common need for parking" Ron Bleed.

As mentioned above, the challenges from the highly-decentralized KU MASC 2018 Research Retreat nature of the institution include many duplicative systems, differing views on what standards individual IT units should follow, little or no consistent expectations of compliance to federal, state, local, or campus laws and policies, multiple data centers of varying capability, significantly differing levels of support (making for a lot of "haves" and even more "have nots"), and on it goes.

Coupled with this, the institution has suffered from budget cuts for several years; some coming from the state cutting state appropriations, and some due to a declining enrollment. Budget cuts have been applied across the board with no strategic application, happening with the expectation that there is no reduction of services with less funding-do more with less. This approach has degraded services, led to an extremely highoperations tempo and the feeling that all efforts were focused on fighting fires. Even though one can shoot from the hip accurately, it cannot be sustained for a long-term. All this with an everincreasing usage of technology on both the academic-side and the business-side of the house.

\section{The Problem}

With the decentralized culture that exists at K-State, it is difficult to provide a standard minimum-level of service. This was not only true in supporting the business parts of the institution (business and finance, student services, enrollment management, facilities, construction and planning, etc.), the teaching and learning environment, and faculty scholarship; it was true in supporting research.

I heard consistently from researchers (PIs, faculty, and scientists) and associate deans for research at all of the 60 
colleges that there was no standard research support. Researchers were left on their own to determine the best approach for managing the data lifecycle (capturing, storing, working with, securing, compliance, and curation) and providing access to high-performance computing. Researchers are spending a significant part of their start-up time figuring out how to handle the research technology effort. In some cases they are building their own environments (many of which do not have the full capabilities to support the research project), individually working with a cloud vendor, or using institutional standard business environments (i.e. administrative network storage - not setup for this purpose).

To clarify, the computer science department does run a high performance computer Beowulf cluster called BeoCat (we are the Wildcats after all). Great custodians of their resources, they make it available to all who ask for no cost (other than frequent requests to add a resource to the grant request here, but this service is informal and not utilized consistently).

\section{What Now?}

Following a framework similar to that identified in the EDUCAUSE Review article Building Research Cyberinfrastructure at Small/Medium Research Institutions, we plan on following a standard strategic planning approach. First, we need to do a full assessment of our current structure. We plan to ask a variety of questions:

- What research technology support do researchers need?

- Assistance with:

- Data Collection

- Storage
- Data Analysis

- Compliance with security expectations (CUI, GDPR, etc.)

- Archiving

- Curation

- High Performance Computing cycles

○ Training

- Scheduling

- Cloud or on premises

- What is working well now?

- What isn't working well?

Working in collaboration with the Provost/Executive Vice President for Academic Affairs and the Vice President for Research, this input will be analyzed and a formal plan will be developed, with prioritized actions. A major component of this plan needs to include support strategies with clear lines drawn on who does what. The development of the cyberinfrastructure needed to support research is a must. The creation of a governance committee with participation from faculty, associate deans of research, members from office of the Vice President for Research and the office of the $\mathrm{CIO}$ will focus on developing and implementing this plan.

Once we have a fully fleshed plan, understanding the costs and developing a strategy for funding must become a focus. This includes a hybrid plan for on premises, cloud-based, and partnership solutions (Agee, A., Rowe, T., Woo, M., Woods, D. (2010). Building Research Cyberinfrastructure at Small/Medium Research Institutions. EDUCAUSEReview).

There is a need to hold researchers accountable to the expectations of the institution and the granting source. Ensuring the academic freedom for 
researchers to follow their own path to a solution, yet providing the basic cyberinfrastructure services and support is an interesting balance.

As we develop the right-sized plan for this cyberinfrastructure, find the funding to implement and sustain it, and have a plan for scaling it to achieve the institution's 2025 goals for research, we "need to find a balance between centralizing IT operations and providing sufficient flexibility and freedom to allow researchers to innovate 'at the edges' in individual departments and laboratories" (Hacker, T. J., Wheeler, B. C. (2007). Making Research Cyberinfrastructure a Strategic Choice. EDUCAUSE Quarterly).

The next few years will be an exciting challenge as we negotiate our path to developing and running the research technology environment for the twentyfirst century. 